\title{
Clinical Manifestation, Timing Course, Precipitating Factors, and Protective Factors of Ventricular Free Wall Rupture Following ST-Segment Elevation Myocardial Infarction
}

\author{
Wei Gong, ${ }^{1,2}$ MD, Han Shi, ${ }^{1,2}$ MD, Mengwen Yan, ${ }^{3}$ MD, Yan Yan, ${ }^{1,2}$ MD, Xiao Wang, ${ }^{1,2}$ MD, \\ Siyi $\mathrm{Li},{ }^{1,2} \mathrm{MD}$, Xiaonan $\mathrm{He},{ }^{1,2} \mathrm{MD}$ and Shaoping Nie, ${ }^{1,2} \mathrm{MD}$
}

\begin{abstract}
Summary
Ventricular free wall rupture (FWR) is a catastrophic complication of that occurs after acute myocardial infarction (AMI), and at present, its clinical characteristics are unclear. We analyzed a total of 6,712 consecutive patients who presented with ST-segment elevation myocardial infarction (STEMI), and 78 patients with FWR after STEMI were enrolled in the study. Patients' demographic data, clinical manifestation, laboratory test results, and angiographic features were then collected and analyzed. FWR occurred in 78 cases $(1.16 \%)$, and the inhospital mortality of FWR was up to $92.3 \%$. Among the 78 FWR patients, 72 obtained accurate rupture time. FWR typically occurred within the first week after the infarct. Compared to late-phase FWR (more than 48 hours after STEMI) patients, early-phase FWR (during 48 hours after STEMI) patients showed significantly higher random glucose and higher percentage of anterior myocardial infarction. Besides, dual antiplatelet therapy (DAPT), $\beta$-blockers, and angiotensin-converting enzyme inhibitors/angiotensin receptor blocker (ACEI/ ARB) were used less frequently in early-phase FWR patients. Moreover, we first reported the precipitating factors of FWR. Defecating, transporting, acute emotional upset, diets, and invasive treatment turned out to be the main triggers for FWR. Furthermore, we found that patients who survived from FWR were younger, had higher $\beta$-blocker coverage in the inhospital treatment, and had a higher frequency of primary PCI. FWR remains an infrequent but devastating complication of STEMI. We have found several factors related to the occurrence and prognosis of FWR. This study provides evidence for a better understanding of FWR.
\end{abstract}

Key words: Acute myocardial infarction, Cardiac rupture, Clinical characteristics

(Int Heart J 2020; 61: 651-657)

$\mathrm{O}$ ver the last decades, the mortality of acute myocardial infarction (AMI) has been decreasing with the development of reperfusion therapy and adjunctive pharmacological therapies. ${ }^{1-3)}$ Unfortunately, the mortality of ventricular free wall rupture (FWR), a catastrophic complication secondary to AMI, remains high. In fact, previous studies have reported mortality of FWR up to $90 \% .^{4 \cdot 6)}$

Due to the rare and unpredictable occurrence of FWR, evidence for FWR clinical characteristics was lacking. Unsurprisingly, there was no consensus on the prevention and treatment of FWR. ${ }^{6-10)}$ FWR is associated with a high mortality from electromechanical dissociation or pericardial tamponade and is often unresponsive to resuscitative maneuver. Our knowledge of FWR is limited to case reports and retrospective studies frequently combining it to ventricular septal rupture (VSR) and/or papillary muscle rupture (PMR) ${ }^{4-6,10-12)}$ However, obviously, differences between FWR and VSR/PMR exist, such as incidence, clinical manifestations, and risk factors. ${ }^{6,11,13)}$ Different from the clinical manifestation of FWR, VSR, and PMR patients often progress to refractory heart failure. . $^{12,13)}$ Nozoe et al. reported that the coronary lesion characteristics and past medical history of FWR patients are markedly different from those of VSR and PMR patients. ${ }^{11)}$ In addition, Honda et al. showed that FWR was a significant determinant for inhospital death in patients with cardiac rupture. ${ }^{5)}$ The clinical features of FWR are still unclear.

This study aims to investigate the characteristics and clinical factors associated with FWR occurrence and prognosis after AMI.

From the ${ }^{1}$ Emergency \& Critical Care Center, Beijing Anzhen Hospital, Capital Medical University, Beijing, China, ${ }^{2}$ Beijing Institute of Heart, Lung, and Blood Vessel Diseases, Beijing, China and ${ }^{3}$ Department of Cardiology, China-Japan Friendship Hospital, Beijing, China.

This study was supported by grants from the National Natural Science Foundation of China (81970292, 81600213, 81670222, and 81700262). Beijing Natural Science Foundation (7191002). Beijing Hospitals Authority Youth Program (QML20190603). Capital's Funds for Health Improvement and Research (2018-1-2061). CS Optimizing Antithrombotic Research Fund (BJUHFCSOARF201901-08). Beijing Municipal Administration of Hospitals Clinical Medicine Development of Special Funding Support (ZYLX201710), Beijing Municipal Administration of Hospitals' Ascent Plan (DFL20180601).

Address for correspondence: Shaoping Nie, MD, Beijing Anzhen Hospital, Capital Medical University, No. 2 Anzhen Road, Chaoyang District, Beijing 100029, China. E-mail: spnie@ccmu.edu.cn

Received for publication October 14, 2019. Revised and accepted February 19, 2020.

Released in advance online on J-STAGE July 18, 2020.

doi: 10.1536/ihj.19-541

All rights reserved by the International Heart Journal Association. 
Table I. Baseline Patient Characteristics

\begin{tabular}{|c|c|c|c|}
\hline Variables & STEMI group $(n=156)$ & FWR group $(n=78)$ & $P$ value \\
\hline Age, years & $57.0[49.0,63.0]$ & $73.0[67.5,79.0]$ & $<0.001$ \\
\hline Female & $21(13.5)$ & $43(55.1)$ & $<0.001$ \\
\hline Smoking & $104(66.7)$ & $24(30.8)$ & $<0.001$ \\
\hline Alcohol & 43 (27.6) & $14(17.9)$ & 0.145 \\
\hline Prior hypertension & $80(51.3)$ & $47(60.3)$ & 0.212 \\
\hline Prior diabetes & $40(25.6)$ & $23(29.5)$ & 0.536 \\
\hline Prior MI & $17(10.9)$ & $6(7.7)$ & 0.494 \\
\hline Anterior MI & $80(51.3)$ & $51(65.4)$ & 0.050 \\
\hline Time from onset to admission (hours) & $6.3[4.0,12.0]$ & $6.0[3.0,11.3]$ & 0.360 \\
\hline $\mathrm{SBP}(\mathrm{mm} \mathrm{Hg})$ & $120.0[110.0,132.0]$ & $115.0[103.0,126.5]$ & 0.013 \\
\hline DBP (mm Hg) & $70.0[70.0,80.0]$ & $70.0[60.0,77.3]$ & 0.171 \\
\hline Heart rate $(\mathrm{bpm})$ & $72.0[65.0,80.0]$ & $79.5[65.0,92.3]$ & 0.004 \\
\hline Killip class III or IV & $16(10.3)$ & $18(23.1)$ & 0.011 \\
\hline $\operatorname{WBC}\left(1,000 / \mathrm{mm}^{3}\right)$ & $10.4 \pm 2.9$ & $11.3 \pm 3.2$ & 0.033 \\
\hline Hemoglobin $(\mathrm{g} / \mathrm{L})$ & $147.0[137.0,158.0]$ & $133.0[121.0,140.0]$ & $<0.001$ \\
\hline Platelet count $\left(1,000 / \mathrm{mm}^{3}\right)$ & $220.0[189.3,252.0]$ & $211.5[170.0,248.5]$ & 0.198 \\
\hline Random glucose $(\mathrm{mmol} / \mathrm{L})$ & $6.3[5.4,8.1]$ & $8.4[6.6,11.5]$ & $<0.001$ \\
\hline Aspirin + thienopyridine & $155(99.4)$ & $72(92.3)$ & 0.006 \\
\hline Anticoagulants & $149(95.5)$ & $71(91.0)$ & 0.241 \\
\hline ACEI/ARB & $111(71.2)$ & $24(30.8)$ & $<0.001$ \\
\hline$\beta$-Blockers & $122(78.2)$ & $41(52.6)$ & $<0.001$ \\
\hline Statin & 148 (94.9) & $65(83.3)$ & 0.006 \\
\hline Primary PCI & $106(67.9)$ & $19(24.3)$ & $<0.001$ \\
\hline Thrombolysis & $5(3.2)$ & $6(7.7)$ & 0.187 \\
\hline
\end{tabular}

Data given as $n(\%)$, mean $\pm \mathrm{SD}$, or median (IQR). ACEI indicates angiotensin-converting enzyme inhibitor; $\mathrm{ARB}$, angiotensin receptor blocker; DBP, diastolic blood pressure; IQR, interquartile range; MI, myocardial infarction; PCI, percutaneous coronary intervention; SD, standard deviation; SBP, systolic blood pressure; and WBC, white blood cell counts.

\section{Methods}

Study patients and design: We analyzed a total of 6,712 consecutive patients who presented with ST-segment elevation myocardial infarction (STEMI) and admitted to Beijing Anzhen Hospital (Beijing, China), from January 2010 to November 2017. This study enrolled a total of 78 patients with FWR after STEMI. STEMI was defined in terms of the following criteria: ongoing ischemic symptoms, typical rise or fall in cardiac biomarkers, and a new ST elevation in two or more contiguous leads with leads $\mathrm{V} 1, \mathrm{~V} 2$, and V3 measuring at least 0.2 or $0.1 \mathrm{mV}$ in the remaining leads or new developed left bundle-branch block pattern. ${ }^{14)}$ The diagnosis of FWR was based on surgery results or clinical manifestations and examination results (echocardiography and diagnostic pericardiocentesis). The clinical diagnosis of FWR was made in those patients with sudden circulatory collapse, electrocardiographic evidence of electromechanical dissociation and bradycardia, and pericardial effusion as shown by echocardiography.

We performed case-control study with the ratio of 1 : 2, and 156 STEMI patients without FWR were randomly selected as the control group, based on the medical record number coding.

All procedures followed were in accordance with the Helsinki Declaration of 1975, as revised in 2008. All patients were given their informed written consent. The study was approved by the ethics committee of Beijing Anzhen Hospital.

Data collection: Baseline demographic data, clinical char- acteristics, patients' medical history, and precipitating factors of FWR were collected from the medical records of the recruited patients. Defecation is defined as the elimination of solid or semisolid waste materials (feces) from the digestive tract. The parameters of the blood test and vital signs (blood pressure, heart rate) were the first test results of the patients after admission.

Statistical analysis: Variables following a normal distribution were expressed as mean \pm standard deviations. Variables following a non-normal distribution were expressed as median (interquartile range). The mean of normally distributed variables were compared using the Student's $t$-test. The Mann-Whitney $U$ test was used for skewed data. For comparisons of the distributions of variables between groups, Chi-square or Fisher exact tests was used. $P<0.05$ was considered to be statistically significant. Statistical analysis was performed with IBM SPSS Statistics, version 22.0 (IBM Corp., Armonk, NY, USA).

\section{Results}

Baseline patient characteristics: In the 6,712 consecutive patients with STEMI, there were 78 identified FWR patients $(1.16 \%)$. Table I shows the baseline patient characteristics. Among the 78 patients with STEMI and FWR, the median age was 73.0 years $[67.5,79.0]$, and $55.1 \%$ were female. Fifty-one patients $(65.4 \%)$ suffered from anterior myocardial infarction. In 19 (24.3\%) patients, infarct-related arteries were opened after primary PCI. 
A

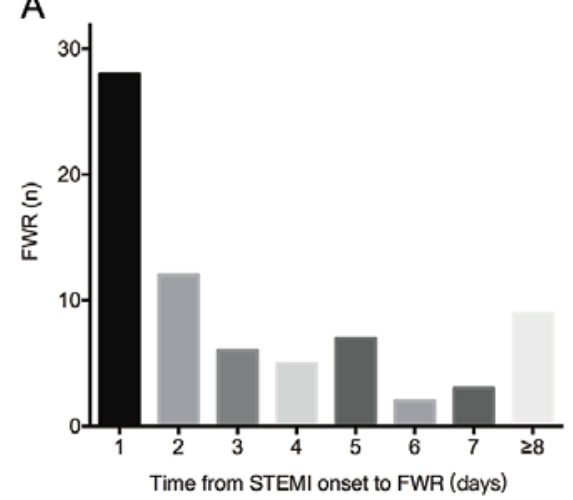

C

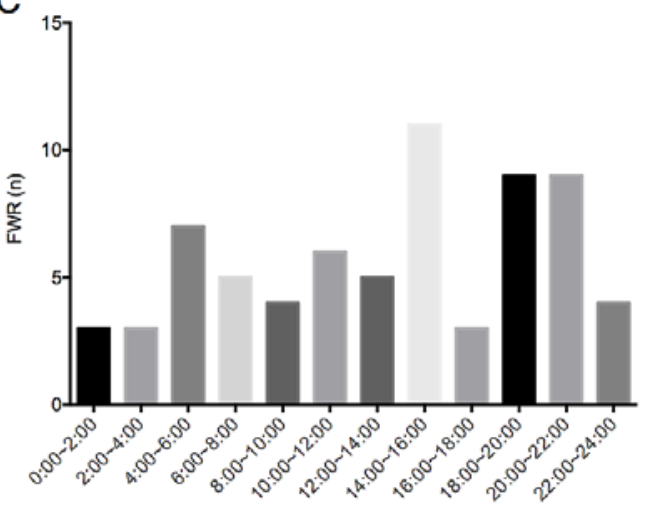

B

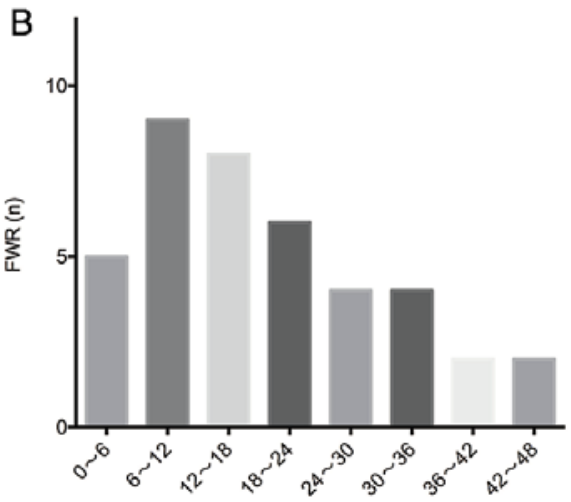

Time from STEMI onset to FWR (h)

Figure 1. Timing course of FWR. A: Time from STEMI onset to FWR. B: Distribution of FWR patients within 48 hours after STEMI onset. C: Distribution of FWR occurring 24 hours a day. FWR, free wall rupture; STEMI, ST-segment elevation myocardial infarction.

Only two patients underwent surgery. Among the 78 FWR patients, $72(92.3 \%)$ died due to myocardial rupturerelated pericardial tamponade in hospital, and the rest of the patients survived at discharge.

Compared to STEMI patients, FWR patients were older and more likely to be women with an anterior infarct, whereas a previous history of smoking was less common (Table I). At admission, FWR patients presented more frequently with higher Killip class, faster heart rate, and lower systolic blood pressure; random glucose and white blood cell counts were significantly higher in the FWR group, whereas hemoglobin levels of the FWR group were significantly lower than the STEMI group. Recommended medications including dual antiplatelet therapy (DAPT), $\beta$-blockers, and angiotensin-converting enzyme inhibitors/angiotensin receptor blocker (ACEI/ ARB) were used less frequently in FWR patients. Furthermore, primary PCI treatment was used less frequently in FWR patients.

Timing course of FWR: Among the 78 FWR patients, 72 obtained accurate rupture time. FWR occurred in 63 $(87.5 \%)$ of the patients within the first week after STEMI and in $40(55.6 \%)$ of the patients during the first 48 hours (Figure 1A). As shown in Figure 1B, 6-24 hours after the onset of STEMI is the peak of FWR occurrence.

Furthermore, FWR occurred in $62.5 \%$ (45/72) of the patients between $5 \mathrm{PM}$ and $8 \mathrm{AM}$, indicating that an im- portant proportion of these events occurred outside the regular office schedule. The peak period of FWR was between 14:00 and 22:00, and FWR occurred in 51.4\% (37/ 72) of the patients during this period (Figure 1C).

Comparison between early- and late-phase FWR: Early-phase FWR (during 48 hours after STEMI) happened in 40 patients $(55.6 \%)$ and that of the late phase (more than 48 hours after STEMI) happened in 32 patients in total. We carried on to investigate the potential factors contributing to FWR in different stages (Table II). Early-phase FWR patients showed significantly higher random glucose $(10.9 \pm 0.8$ versus $8.7 \pm 0.7 \mathrm{mmol} / \mathrm{L}, P=$ $0.039)$ than, while the percentage of anterior myocardial infarction is higher $(75 \%$ versus $50 \%, P=0.026)$. Besides, DAPT, $\beta$-blockers and ACEI/ARB were used less frequently in early-phase FWR patients. In addition, there was a trend that patients with early-phase FWR are more likely to be women with faster heart rate.

Thus, we further investigated the relationship between different phases and these potential factors and found that random glucose weakly correlated with the time to rupture after STEMI ( $r=-0.265, P=0.027$ ) (Figure 2A). Additionally, the time from the onset of STEMI to FWR was significantly shortened in patients with random blood glucose greater than $10 \mathrm{mmol} / \mathrm{L}$ (Figure 2B).

Precipitating factors of FWR: Among the 78 FWR patients, $29(37.2 \%)$ were traced to a clear rupture- 
Table II. Comparison between Early-Phase and Late-Phase FWR

\begin{tabular}{|c|c|c|c|}
\hline Variables & $\begin{array}{c}\text { Early-phase FWR } \\
(n=40)\end{array}$ & $\begin{array}{c}\text { Late-phase FWR } \\
\qquad(n=32)\end{array}$ & $P$ value \\
\hline Age, years & $73.7 \pm 1.5$ & $73.0 \pm 1.3$ & 0.759 \\
\hline Female & $26(65.0)$ & $14(43.7)$ & 0.059 \\
\hline Smoking & $11(27.5)$ & $12(37.5)$ & 0.448 \\
\hline Alcohol & $8(20)$ & $4(12.5)$ & 0.529 \\
\hline Prior hypertension & $22(55.0)$ & $21(65.6)$ & 0.251 \\
\hline Prior diabetes & $15(37.5)$ & 7 (21.9) & 0.138 \\
\hline Prior MI & $3(7.5)$ & $3(9.4)$ & 0.551 \\
\hline Anterior myocardial infarction & $30(75.0)$ & $16(50.0)$ & 0.026 \\
\hline Time from onset to admission (hours) & $6.0[3.0,10.8]$ & $6.0[3.3,14.5]$ & 0.397 \\
\hline $\mathrm{SBP}(\mathrm{mm} \mathrm{Hg})$ & $120.0[100.0,140.0]$ & $113.0[105.0,127.3]$ & 0.606 \\
\hline DBP (mm Hg) & $70.0[60.0,75.0]$ & $71.0[67.0,79.5]$ & 0.327 \\
\hline Heart rate $(\mathrm{bpm})$ & $83.0[68.0,96.0]$ & $72.0[61.0,84.0]$ & 0.060 \\
\hline Killip class III or IV & $10(25.0)$ & $7(21.9)$ & 0.490 \\
\hline $\operatorname{WBC}\left(1,000 / \mathrm{mm}^{3}\right)$ & $11.5 \pm 0.5$ & $10.4 \pm 0.6$ & 0.128 \\
\hline Hemoglobin $(\mathrm{g} / \mathrm{L})$ & $126.7 \pm 3.1$ & $134.5 \pm 2.4$ & 0.056 \\
\hline Platelet count $\left(1,000 / \mathrm{mm}^{3}\right)$ & $216.5 \pm 10.0$ & $210.1 \pm 10.8$ & 0.665 \\
\hline Random glucose $(\mathrm{mmol} / \mathrm{L})$ & $10.9 \pm 0.8$ & $8.7 \pm 0.7$ & 0.039 \\
\hline Aspirin + thienopyridine & $36(90.0)$ & $30(93.8)$ & 0.449 \\
\hline Anticoagulants & $34(85.0)$ & $30(93.8)$ & 0.103 \\
\hline ACEI/ARB & $8(20.0)$ & $13(40.6)$ & 0.049 \\
\hline$\beta$-Blockers & $13(32.5)$ & $23(71.9)$ & 0.001 \\
\hline Satin & $32(80)$ & $28(87.5)$ & 0.529 \\
\hline Primary PCI & $6(15.0)$ & $8(25.0)$ & 0.222 \\
\hline Thrombolysis & $3(7.5)$ & $2(6.3)$ & 0.606 \\
\hline Time from onset to FWR (hours) & $19.3 \pm 1.9$ & $129.8 \pm 11.1$ & $<0.001$ \\
\hline
\end{tabular}

Data given as $n(\%)$, mean $\pm \mathrm{SD}$, or median (IQR). ACEI indicates angiotensin-converting enzyme inhibitor; $\mathrm{ARB}$, angiotensin receptor blocker; DBP, diastolic blood pressure; IQR, interquartile range; MI, myocardial infarction; PCI, percutaneous coronary intervention; SD, standard deviation; SBP, systolic blood pressure; and WBC, white blood cell counts.

A

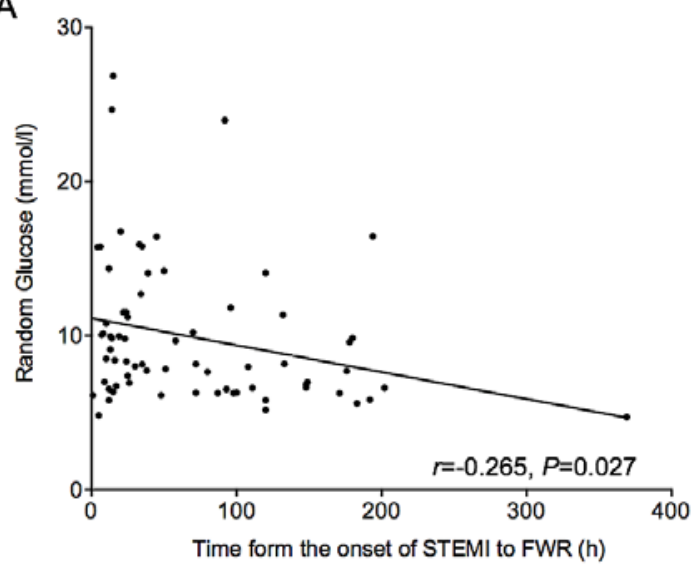

B

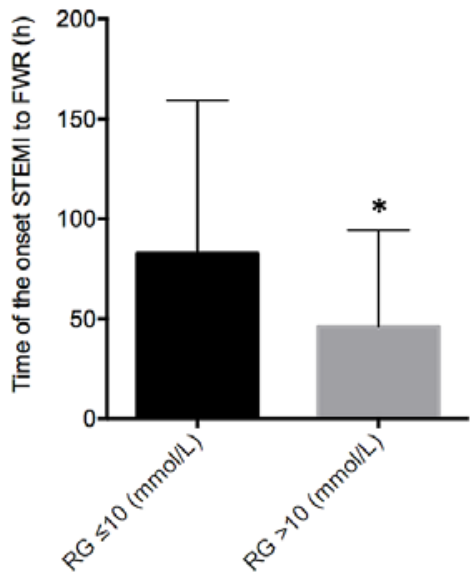

Figure 2. The relationship between random glucose and the time to rupture after STEMI. A: Random glucose weakly correlated with the time to rupture after STEMI. B: The time from the onset of STEMI to FWR was significantly shortened in patients with random glucose greater than $10 \mathrm{mmol} / \mathrm{L}$. FWR, free wall rupture; RG, random glucose; STEMI, ST-segment elevation myocardial infarction.

precipitating factor. Defecating (11 patients), transporting (5 patients), acute emotional upset (3 patients), diets (3 patients), invasive treatment ( 2 patients), and sudden cough ( 2 patients) were the main triggers for FWR. Furthermore, getting up in the morning and washing before bed were also triggers for FWR.
The relationship between clinical factors and inhospital mortality of FWR: Furthermore, we investigated the predictors for inhospital mortality of FWR. We divided patients into inhospital death $(n=72,92.3 \%)$ and inhospital alive $(n=6,7.7 \%)$ groups (Table III). Compared to the alive group, inhospital death group was older $(73.1 \pm 9.0$ 
Table III. The Relationship between Clinical Factors and Inhospital Mortality of FWR

\begin{tabular}{|c|c|c|c|}
\hline Variables & $\begin{array}{l}\text { Inhospital Death } \\
\quad(n=72)\end{array}$ & $\begin{array}{l}\text { Inhospital Alive } \\
\qquad(n=6)\end{array}$ & $P$ value \\
\hline Age, years & $73.1 \pm 9.0$ & $60.3 \pm 11.2$ & 0.002 \\
\hline Female & $40(55.6)$ & $3(50.0)$ & 1.000 \\
\hline Smoking & $23(31.9)$ & $1(16.7)$ & 0.660 \\
\hline Alcohol & $13(18.1)$ & $1(16.7)$ & 1.000 \\
\hline Prior hypertension & $43(59.7)$ & $4(66.7)$ & 1.000 \\
\hline Prior diabetes & $22(30.6)$ & $1(16.7)$ & 0.663 \\
\hline Prior MI & $6(8.3)$ & $0(0)$ & 1.000 \\
\hline Anterior myocardial infarction & $46(63.9)$ & $5(83.3)$ & 0.658 \\
\hline Time from onset to admission (hours) & $6.0[3.0,12.0]$ & $4.0[1.8,6.0]$ & 0.110 \\
\hline $\mathrm{SBP}(\mathrm{mm} \mathrm{Hg})$ & $116.0[103.0,128.0]$ & $115.0[102.5,125.3]$ & 0.834 \\
\hline $\mathrm{DBP}(\mathrm{mm} \mathrm{Hg})$ & $70.0[61.0,78.0]$ & $70.0[60.0,74.5]$ & 0.702 \\
\hline Heart rate (bpm) & $83.0[68.0,96.0]$ & $72.0[61.0,84.0]$ & 0.482 \\
\hline Killip class III or IV & $18(25.0)$ & $0(0.0)$ & 0.327 \\
\hline WBC $\left(1,000 / \mathrm{mm}^{3}\right)$ & $11.4 \pm 3.2$ & $10.3 \pm 3.5$ & 0.427 \\
\hline Hemoglobin (g/L) & $130.1 \pm 17.0$ & $138.2 \pm 19.9$ & 0.272 \\
\hline Platelet count $\left(1,000 / \mathrm{mm}^{3}\right)$ & $214.2 \pm 60.8$ & $172.5 \pm 32.4$ & 0.102 \\
\hline Random glucose $(\mathrm{mmol} / \mathrm{L})$ & $10.2 \pm 5.0$ & $9.1 \pm 3.7$ & 0.610 \\
\hline Aspirin + thienopyridine & $66(91.7)$ & $6(100.0)$ & 1.000 \\
\hline Anticoagulants & $65(90.3)$ & $6(100.0)$ & 1.000 \\
\hline ACEI/ARB & $20(27.8)$ & $4(66.7)$ & 0.069 \\
\hline$\beta$-Blockers & 35 (48.6) & $6(100.0)$ & 0.027 \\
\hline Satin & $59(81.9)$ & $6(100.0)$ & 0.582 \\
\hline Primary PCI & $14(19.4)$ & $5(83.3)$ & 0.003 \\
\hline Thrombolysis & $5(6.9)$ & $1(16.7)$ & 0.392 \\
\hline
\end{tabular}

Data given as $n(\%)$, mean $\pm \mathrm{SD}$, or median (IQR). ACEI indicates angiotensin-converting enzyme inhibitor; $\mathrm{ARB}$, angiotensin receptor blocker; DBP, diastolic blood pressure; IQR, interquartile range; MI, myocardial infarction; PCI, percutaneous coronary intervention; SD, standard deviation; SBP, systolic blood pressure; and WBC, white blood cell counts.

versus $60.3 \pm 11.2, P=0.002$ ). Furthermore, the utilization percentage of $\beta$-blocker was significantly lower in the death group $(48.6 \%$ versus $100 \%, P=0.027)$, and primary PCI treatment was used less frequent in inhospital death patients $(19.4 \%$ versus $83.3 \%, P=0.003)$.

\section{Discussion}

FWR complicating STEMI represented a relatively rare but extremely dangerous event. In this observational study, we found FWR occurred in 78 cases $(1.16 \%)$ and its inhospital mortality was up to $92.3 \%$. FWR typically occurred within the first 48 hours after the infarct (earlyphase FWR). Early-phase FWR patients had significantly higher random glucose; higher proportion of anterior myocardial infarction; and lower use of DAPT, $\beta$-blockers, and ACEI/ARB than the late-phase FWR patients. Patients who survived from FWR were younger and had higher $\beta$ blocker coverage and higher frequency of primary PCI. Moreover, we first reported the precipitating factors of FWR. This study provides evidence for a better understanding of FWR. Previous studies reported that the overall incidence of FWR after AMI ranges from $0.5 \%$ to $6.2 \% .^{6,13,15,16)}$ The FWR rate was $1.16 \%$ in our study. The incidence of FWR reported in different studies was different, which may be due to the following reasons: the diagnosis of FWR mainly depended on autopsy and the autopsy rate varied in different studies; with the development of reperfusion therapy and adjuvant drug therapy, the incidence of FWR has changed. There was still lack of accurate, fast, and effective methods for diagnosing FWR. We held the idea that the incidence of FWR may be underestimated; for instance, pericardial effusion in patients after STEMI may be caused by FWR but was misdiagnosed due to the lack of accurate diagnostic methods.

Although the incidence of FWR is low, FWR has extremely poor clinical prognosis. According to statistical data, the hospital mortality of FWR was up to $90 \%$ or more. ${ }^{4-6)}$ In our study, the inhospital mortality of FWR was up to $92.3 \%$. Because of its extremely dangerous clinical manifestation, clinical treatment is facing great difficulties, and there is no effective treatment. Only a few retrospective studies suggested that emergency surgery could improve the clinical outcome of FWR. ${ }^{17,18)}$ However, surgical treatment also faces great difficulties: sudden death occurred in most FWR patients and there is no time window for surgical treatment. Due to the lack of fast and accurate diagnostic methods, some patients failed to confirm the diagnosis in time and missed the timing of surgery. Figueras et al. found that only $21.1 \%$ of patients diagnosed with cardiac rupture underwent surgery. ${ }^{6}$ Unfortunately, only two patients underwent surgery in this study. Surgical risk, operating difficulty, and postoperative mortality were high. A single-center observational study showed that inhospital mortality was still as high as $33.3 \%$ after emergency surgery in FWR patients. ${ }^{18)}$ Therefore, finding effective interventions to prevent the occurrence of cardiac rupture is key to future research direc- 
tion.

FWR is hazardous, and clinical treatment is limited. At present, there is no effective means of treatment. Therefore, early prevention of the occurrence of FWR is crucial. This study found that FWR occurred in $87.5 \%$ of the patients within the first week after STEMI and in $55.6 \%$ of the patients during the first 48 hours, suggesting that enhanced monitor in the early stages of STEMI (within 48 hours) was important. Furthermore, between 14:00 and 22:00 is high-risk period for FWR, and FWR occurred in $51.4 \%$ of the patients during this period, which might related to hormonal changes in patients and activities in which patients participated. In this study, FWR patients were divided into early- and late-phase groups according to the time from STEMI onset to FWR. In this observational study, we found that early-phase FWR (during 48 hours after STEMI) happened in $40 \mathrm{pa}-$ tients $(55.6 \%)$. Random glucose; anterior myocardial infarction; and utilization percentage of DAPT, $\beta$-blockers, and ACEI/ARB were correlated with early-phase FWR. The results provide evidence for early prevention of FWR. However, further investigations based on larger cohorts are warranted.

In this study, we first reported the precipitating factors of FWR. We found that defecation, patient transfer, and emotional fluctuation were important inducement of FWR, with defecation accounting for $37.9 \%$ of all inducement. Previous studies showed that defecation was associated with sudden cardiac death, ${ }^{19,20)}$ and the possible underlying mechanisms were that patients often held breath when going to stool, especially those with constipation; under this circumstance, the pectoralis, abdominal muscles, and diaphragm contract, and the pressures of thoracic cavity and abdominal cavity increase. The sympathetic nerves also excite. These physiological changes caused significant increase in cardiac load and accelerated contraction of the heart. Our study suggests that STEMI patients should maintain a smooth bowel movement and take laxative treatment if necessary, as a means of reducing the incidence of FWR.

The prognosis of FWR is very poor due to its unpredictable nature and terrible clinical outcomes. Some studies reported that the prognosis of cardiac rupture is dependent on a number of factors; which portion of the myocardium is involved in the rupture, including how long after cardiac rupture occurs; and the treatment that patients receive. ${ }^{21,22)}$ However, as the sample sizes are all relatively limited in the previous studies, the relationship between these factors and prognosis of FWR remains undefined. In this observational study, we have also found that patients who survived from FWR were younger, had higher $\beta$-blocker coverage in the inhospital treatment, and had a higher frequency of primary PCI.

Several limitations exist in this clinical research. First, this was a retrospective observational study; therefore, some information is subject to certain inherent limitation and potential biases, including collection of nonrandomized data and missing or incomplete information. Second, FWR is an uncommon clinical condition and thus, involves a limited number of cases. A further limitation represents undetected cases of "minor" FWR that might alter the overall picture of our results; incomplete rupture could easily be missed. ${ }^{23)}$ On the other hand, FWR may suddenly occur in some patients before admission or after discharge, and this part of the population were unavailable. The incidence of this complication could have been underestimated in our study.

\section{Conclusion}

FWR complicating STEMI represented a relatively rare but highly fatal event. The incidence of FWR resulting from STEMI in our study was $1.16 \%$, and the inhospital mortality of FWR was up to $92.3 \%$. We found that early-phase FWR (during 48 hours after STEMI) patients had significantly higher random glucose; higher proportion of anterior myocardial infarction; and lower use of DAPT, $\beta$-blockers, and ACEI/ARB. In addition, the study showed that patients who survived from FWR was younger, had higher $\beta$-blocker coverage in the inhospital treatment, and had a higher frequency of primary PCI. This study provides evidence for a better understanding of FWR.

\section{Acknowledgment}

The authors thank all the study investigators, staff, and patients.

\section{Disclosure}

Conflicts of interest: The authors declare no potential conflict of interests.

\section{References}

1. Sulo G, Igland J, Sulo E, et al. Mortality following first-time hospitalization with acute myocardial infarction in Norway, 2001-2014: Time trends, underlying causes and place of death. Int J Cardiol 2019; 294: 6-12.

2. Yamamoto T, Yoshida N, Takayama M, Tokyo CCU Network Temporal trends in acute myocardial infarction incidence and mortality between 2006 and 2016 in Tokyo-report from the Tokyo CCU Network. Circ J 2019; 83: 1405-9.

3. Hashimoto T, Ako J, Nakao K, et al. Pre-procedural thrombolysis in myocardial infarction flow in patients with ST-segment elevation myocardial infarction. Int Heart J 2018; 59: 920-5.

4. López-Sendón J, Gurfinkel EP, Lopez de Sa E, et al. Factors related to heart rupture in acute coronary syndromes in the Global Registry of Acute Coronary Events. Eur Heart J 2010; 31: 1449-56.

5. Honda S, Asaumi Y, Yamane T, et al. Trends in the clinical and pathological characteristics of cardiac rupture in patients with acute myocardial infarction over 35 years. J Am Heart Assoc 2014; 3: e000984.

6. Figueras J, Alcalde O, Barrabés JA, et al. Changes in hospital mortality rates in 425 patients with acute ST-elevation myocardial infarction and cardiac rupture over a 30 -year period. Circulation 2008; 118: 2783-9.

7. Gong W, Feng S, Wang X, Fan J, Li A, Nie SP. Beta-blockers reduced the risk of cardiac rupture in patients with acute myocardial infarction: A meta-analysis of randomized control trials. Int J Cardiol 2017; 232: 171-5.

8. Matteucci M, Fina D, Jiritano F, et al. Treatment strategies for 
post-infarction left ventricular free-wall rupture. Eur Heart J Acute Cardiovasc Care 2019; 8: 379-87.

9. Tang L, Tang JJ, Hu XQ, et al. Transcatheter closure of complex post-myocardial infarction left ventricular pseudoaneurysm and unique post-traumatic right ventricular pseudoaneurysm. Int Heart J 2019; 60: 998-1002.

10. Matsumura K, Kin H, Matsuki R, et al. Cardiac rupture due to reinfarction in the early phase of apical myocardial infarction. Int Heart J 2019; 60: 974-8.

11. Nozoe M, Sakamoto T, Taguchi E, Miyamoto S, Fukunaga T, Nakao K. Clinical manifestation of early phase left ventricular rupture complicating acute myocardial infarction in the primary PCI era. J Cardiol 2014; 63: 14-8.

12. Crenshaw BS, Granger CB, Birnbaum Y, et al. Risk factors, angiographic patterns, and outcomes in patients with ventricular septal defect complicating acute myocardial infarction. GUSTOI (Global Utilization of streptokinase and TPA for Occluded Coronary arteries) Trial Investigators. Circulation 2000; 101: 27-32.

13. Kutty RS, Jones N, Moorjani N. Mechanical complications of acute myocardial infarction. Cardiol Clin 2013; 31: 519-31.

14. Ibanez B, James S, Agewall S, et al; ESC Scientific Document Group. ESC Guidelines for the management of acute myocardial infarction in patients presenting with ST-segment elevation: The Task Force for the management of acute myocardial infarction in patients presenting with ST-segment elevation of the European Society of Cardiology (ESC). Eur Heart J 2018; 39: 119-77.

15. Qian G, Wu C, Chen YD, Tu CC, Wang JW, Qian YA. Predictive factors of cardiac rupture in patients with ST-elevation myocardial infarction. J Zhejiang Univ Sci B 2014; 15: 1048-
54.

16. Bajaj A, Sethi A, Rathor P, Suppogu N, Sethi A. Acute complications of myocardial infarction in the current era: Diagnosis and management. J Investig Med 2015; 63: 844-55.

17. Haddadin S, Milano AD, Faggian G, et al. Surgical treatment of postinfarction left ventricular free wall rupture. J Card Surg 2009; 24: 624-31.

18. Flajsig I, Castells y, Cuch E, et al. Surgical treatment of left ventricular free wall rupture after myocardial infarction: Case series. Croat Med J 2002; 43: 643-8.

19. Hayashi S, Toyoshima H, Tanabe N, et al. Activity immediately before the onset of non-fatal myocardial infarction and sudden cardiac death. Jpn Circ J 1996; 60: 947-53.

20. Kouno A, Matoba R, Shikata I. A statistical study of sudden cardiac death for past five years in Osaka medical, investigated at the Osaka Medical Examiner's Office. Acta Med Leg Soc (Liege) 1989; 39: 205-15.

21. Lou B, Luo Y, Hao X, et al. Clinical characteristics and protective factors in patients with acute myocardial infarction undergoing in-hospital myocardial free wall rupture: A single-center, retrospective analysis. J Investig Med 2019; 67: 1097-102.

22. Lemery R, Smith HC, Giuliani ER, Gersh BJ. Prognosis in rupture of the ventricular septum after acute myocardial infarction and role of early surgical intervention. Am J Cardiol 1992; 70: $147-51$.

23. Figueras J, Cortadellas J, Domingo E, Soler-Soler J. Survival following self-limited left ventricular free wall rupture during myocardial infarction. Management differences between patients with or without pseudoaneurysm formation. Int J Cardiol 2001; 79: 103-11. 